PROCEEDINGS OF THE

AMERICAN MATHEMATICAL SOCIETY

Volume 131, Number 7, Pages 2235-2240

S 0002-9939(02)06753-9

Article electronically published on October 18, 2002

\title{
THE REMOVAL OF $\pi$ FROM SOME UNDECIDABLE PROBLEMS INVOLVING ELEMENTARY FUNCTIONS
}

\author{
M. LACZKOVICH \\ (Communicated by Carl G. Jockusch, Jr.)
}

\begin{abstract}
We show that in the ring generated by the integers and the functions $x, \sin x^{n}$ and $\sin \left(x \cdot \sin x^{n}\right)(n=1,2, \ldots)$ defined on $\mathbf{R}$ it is undecidable whether or not a function has a positive value or has a root. We also prove that the existential theory of the exponential field $\mathbf{C}$ is undecidable.
\end{abstract}

1.

Let $\mathcal{S}$ denote the class of expressions generated by the rational numbers and $\pi$, the variable $x$, the operations of addition, multiplication, and composition, and the sine function. It was proved by P. S. Wang [5] (based on the papers of D. Richardson [4] and B. F. Caviness [1]) that the existential theory of $\mathcal{S}$ is undecidable. More precisely, Wang proved that for $f \in \mathcal{S}$ each of the following statements is recursively undecidable: (i) there exists a real number $x$ such that $f(x)>0$; and (ii) there exists a real number $x$ such that $f(x)=0$.

Let $\mathcal{S}_{1}$ denote the ring generated by the integers and by the expressions $x, \sin x^{n}$ and $\sin \left(x \cdot \sin x^{n}\right)(n=1,2, \ldots)$. In other words, $\mathcal{S}_{1}$ is the set of expressions obtained by substituting $x, \sin x^{n}$ and $\sin \left(x \cdot \sin x^{n}\right)$ into an arbitrary polynomial with integer coefficients.

In this note we show that in Wang's theorems the class $\mathcal{S}$ can be replaced by $\mathcal{S}_{1}$. This improves Wang's result in two ways: it eliminates the use of $\pi$, and reduces the number of compositions.

Theorem 1. For $f \in \mathcal{S}_{1}$, each of the following statements is recursively undecidable: (i) there exists a real number $x$ such that $f(x)>0$; and (ii) there exists a real number $x$ such that $f(x)=0$.

The proof, which follows Richardson's original argument [4] with some modifications, will be given in the next section.

Let $\mathcal{S}_{2}$ denote the ring generated by the integers and by the expressions $\sin x^{n}$ and $\cos x^{n}(n=1,2, \ldots)$. As the following theorem shows, the statement of Theorem 1 is not very far from being optimal.

Received by the editors February 7, 2002 and, in revised form, February 22, 2002.

2000 Mathematics Subject Classification. Primary 03B25, 03D40; Secondary 26A09.

Key words and phrases. Undecidable problems, rings of elementary functions.

This research was partially supported by the Hungarian National Foundation for Scientific Research Grant No. T032042. 
Theorem 2. There is an algorithm that decides, for every $f \in \mathcal{S}_{2}$, whether or not there exists a real number $x$ such that $f(x)>0$.

Proof. Let $P\left(x_{1}, \ldots, x_{n}, y_{1}, \ldots, y_{n}\right) \in \mathbf{Z}\left[x_{1}, \ldots, x_{n}, y_{1}, \ldots, y_{n}\right]$, and put

$$
Q(x)=P\left(\sin x, \ldots, \sin x^{n}, \cos x, \ldots, \cos x^{n}\right) .
$$

Let $A_{P}$ denote the statement

$$
\begin{gathered}
\exists\left(x_{1}, \ldots, x_{n}, y_{1}, \ldots, y_{n}\right) \in \mathbf{R}^{2 n} \text { such that } x_{i}^{2}+y_{i}^{2}=1(i=1, \ldots, n) \\
\text { and } P\left(x_{1}, \ldots, x_{n}, y_{1}, \ldots, y_{n}\right)>0 .
\end{gathered}
$$

We claim that $\exists x(Q(x)>0) \Longleftrightarrow A_{P}$. Indeed, $\exists x(Q(x)>0) \Longrightarrow A_{P}$ is obvious. Suppose $A_{P}$, and let $x_{i}, y_{i}$ be such that $x_{i}^{2}+y_{i}^{2}=1(i=1, \ldots, n)$ and $P\left(x_{1}, \ldots, x_{n}, y_{1}, \ldots, y_{n}\right)>0$. It is well-known that the sequence

$$
\left(\left\{\frac{k}{2 \pi}\right\},\left\{\frac{k^{2}}{2 \pi}\right\}, \ldots,\left\{\frac{k^{n}}{2 \pi}\right\}\right)(k=1,2, \ldots)
$$

is uniformly distributed in $[0,1]^{n}$. (See [2, Theorem 6.3 on p. 48 and Theorem 3.2 on p. 27].) In particular, this sequence is everywhere dense in $[0,1]^{n}$. Choose $\alpha_{i}$ such that $\left(x_{i}, y_{i}\right)=\left(\sin \alpha_{i}, \cos \alpha_{i}\right)$ for every $i=1, \ldots, n$. Then, for every $\varepsilon>0$, we can select an integer $k$ such that $\left|k^{i}-\alpha_{i}-2 m_{i} \pi\right|<\varepsilon(i=1, \ldots, n)$, where $m_{0}, \ldots, m_{n}$ are also integers. Clearly, if $\varepsilon$ is chosen small enough, then $Q(k)>0$ will be satisfied. By Tarski's theorem, there is an algorithm that decides the truth of $A_{P}$ for every $P \in \mathbf{Z}\left[x_{1}, \ldots, x_{n}, y_{1}, \ldots, y_{n}\right]$. Then the same algorithm decides whether or not $P\left(\sin x, \ldots, \sin x^{n}, \cos x, \ldots, \cos x^{n}\right)>0$ holds for a suitable real number $x$.

Question. Does there exist an algorithm that decides, for every $f \in \mathcal{S}_{2}$, whether or not $f$ has a real root? Does there exist such an algorithm for the ring generated by $\sin x$ and $\sin x^{2}$ ?

The problem of eliminating $\pi$ also appears in the context of the undecidability of the exponential field C. In [3, Theorem 20, p. 201] A. J. Macintyre states that the existential theory of the exponential field $\mathbf{C}$, with $\pi$ as a distinguished constant, is undecidable. Then he poses the problem of removing the use of $\pi$. Unfortunately, Theorem 1 above does not solve this problem, because the notion of real numbers cannot be formulated in $\mathbf{C}$. As Macintyre remarks, the theory of the rationals can be reduced to $\mathbf{C}$. Indeed, we have

$$
x \in \mathbf{Q} \Longleftrightarrow \exists u \exists v\left[\left(e^{u}=e^{v}=1\right) \wedge(v \neq 0) \wedge(v x=u)\right] .
$$

Now the theory of $\mathbf{Q}$ is undecidable by a well-known theorem of J. Robinson. However, the undecidable scheme of statements obtained from J. Robinson's theorem contains universal quantifiers, and the question of whether or not the existential theory of $\mathbf{Q}$ is undecidable seems to be open.

Still, we can solve the problem posed by Macintyre using the simple observation that a rational number $x$ is an integer if and only if $2^{x}$ is rational.

Theorem 3. The existential theory of the exponential field $\mathbf{C}$ is undecidable.

Proof. We claim that for every $x \in \mathbf{Q}$,

$$
x \in \mathbf{Z} \Longleftrightarrow \exists z\left[\left(e^{z}=2\right) \wedge\left(e^{z x} \in \mathbf{Q}\right)\right] .
$$


Indeed, the formula on the right-hand side is true if and only if

$$
e^{(\log 2+2 k \pi i) x}=2^{x}(\cos 2 k \pi x+i \cdot \sin 2 k \pi x)
$$

is rational for a suitable integer $k$. But this happens if and only if $\sin 2 k \pi x=0$ and $2^{x} \cdot \cos 2 k \pi x= \pm 2^{x}$ is rational; that is, when $x$ is an integer.

By the Davis-Putnam-Robinson-Matijasevic theorem, there exists a polynomial $P\left(y, x_{1}, \ldots, x_{n}\right)$ with integer coefficients such that the set

$$
H=\left\{y \in \mathbf{Z}: \exists\left(k_{1}, \ldots, k_{n}\right) \in \mathbf{Z}^{n}\left(P\left(y, k_{1}, \ldots, k_{n}\right)=0\right)\right\}
$$

is not recursive. We shall use the abbreviations

$$
R(x, u, v)=\left[\left(e^{u}=e^{v}=1\right) \wedge(v \neq 0) \wedge(v x=u)\right]
$$

and

$$
I(x, u, v, z)=\left[\left(e^{z}=2\right) \wedge R\left(e^{z x}, u, v\right)\right] .
$$

We put $x=\left(x_{1}, \ldots, x_{n}\right)$, etc., and denote by $Q(y, x, u, v, s, t, z)$ the expression

$$
\begin{aligned}
& R\left(x_{1}, u_{1}, v_{1}\right) \wedge \ldots \wedge R\left(x_{n}, u_{n}, v_{n}\right) \\
& \quad \wedge I\left(x_{1}, s_{1}, t_{1}, z_{1}\right) \wedge \ldots \wedge I\left(x_{n}, s_{n}, t_{n}, z_{n}\right) \wedge\left(P\left(y, x_{1}, \ldots, x_{n}\right)=0\right) .
\end{aligned}
$$

Then $Q$ is an expression of the exponential field $\mathbf{C}$ for every fixed integer $y$. Clearly, the formula $\exists x \exists u \exists v \exists s \exists t \exists z Q$ is true if and only if there are integers $x_{1}, \ldots, x_{n}$ such that $P\left(y, x_{1}, \ldots, x_{n}\right)=0$; that is, when $y \in H$. Since $H$ is not recursive, it follows that no algorithm can decide whether an existential formula in $\mathbf{C}$ is true or not.

\section{Proof of Theorem 1}

By the Davis-Putnam-Robinson-Matijasevic theorem, there exists a polynomial $P\left(y, x_{1}, \ldots, x_{n}\right)$ with integer coefficients such that the set

$$
H=\left\{y \in \mathbf{Z}: \exists\left(k_{1}, \ldots, k_{n}\right) \in \mathbf{Z}^{n}\left(P\left(y, k_{1}, \ldots, k_{n}\right)=0\right)\right\}
$$

is not recursive. Let $Q=Q\left(y, x_{0}, x_{1}, \ldots, x_{n}\right) \in \mathbf{Z}\left[y, x_{0}, x_{1}, \ldots, x_{n}\right]$ be a polynomial such that $Q$ is homogeneous in $x_{0}, x_{1}, \ldots, x_{n}$ and satisfies

$$
Q\left(y, 1, x_{1}, \ldots, x_{n}\right)=P\left(y, x_{1}, \ldots, x_{n}\right) .
$$

We shall denote by $d$ the total degree of $x_{0}, \ldots, x_{n}$ in $Q$. We fix a positive integer $N$ such that

$$
(\pi-3)^{N}<\frac{1}{2 \cdot(n+1)}
$$

and put

$$
S=Q^{2}+\left(x_{0}-3\right)^{2 N} \text { and } \frac{\partial S}{\partial x_{i}}=h_{i} \quad(i=0,1, \ldots, n) .
$$

Lemma 1. For every $i=0,1, \ldots, n$ there exists a $g_{i} \in \mathbf{Z}\left[y, x_{0}, \ldots, x_{n}\right]$ such that $g_{i} \geq 1$ everywhere on $\mathbf{R}^{n+2}$, and for every $\left(y, x_{0}, \ldots, x_{n}\right) \in \mathbf{R}^{n+2}$ and $\left(t_{0}, \ldots, t_{n}\right) \in$ $\mathbf{R}^{n+1}$ with $\left|t_{i}\right| \leq 2(i=0, \ldots, n)$ we have

$$
\left|h_{i}\left(y, x_{0}+t_{0}, \ldots, x_{n}+t_{n}\right)\right| \leq g_{i}\left(y, x_{0}, \ldots, x_{n}\right) \quad(i=0, \ldots, n) .
$$

Proof. It is easy to check that if $h_{i}=\sum c \cdot y^{\alpha} x_{0}^{\beta_{0}} \cdots x_{n}^{\beta_{n}}$, then

$$
g_{i}=1+\sum|c| \cdot\left(y^{2}+1\right)^{\alpha}\left(x_{0}^{2}+3\right)^{\beta_{0}} \cdots\left(x_{n}^{2}+3\right)^{\beta_{n}}
$$

satisfies the requirements. 
Now we define

$$
F\left(y, x_{0}, \ldots, x_{n}\right)=4(n+1)^{2}\left[S+\sum_{i=0}^{n} g_{i}^{2} \cdot \sin ^{2} x_{i}\right] .
$$

Lemma 2. $\left\{y \in \mathbf{Z}: \exists\left(x_{0}, \ldots, x_{n}\right) \in \mathbf{R}^{n+1}\left(F\left(y, x_{0}, \ldots, x_{n}\right)<1\right)\right\}=\{y \in \mathbf{Z}$ : $\left.\exists\left(x_{0}, \ldots, x_{n}\right) \in \mathbf{R}^{n+1}\left(F\left(y, x_{0}, \ldots, x_{n}\right) \leq 1\right)\right\}=H$.

Proof. Let $y \in H$, and let $\left(k_{1}, \ldots, k_{n}\right) \in \mathbf{Z}^{n}$ be such that $P\left(y, k_{1}, \ldots, k_{n}\right)=0$. Then, putting $x_{0}=\pi$ and $x_{i}=\pi k_{i}(i=1, \ldots, n)$ we have, by (1) and (2),

$$
\begin{aligned}
F\left(y, x_{0}, \ldots, x_{n}\right) & =4(n+1)^{2}\left[Q^{2}\left(y, \pi, \pi k_{1}, \ldots, \pi k_{n}\right)+(\pi-3)^{2 N}\right] \\
& =4(n+1)^{2} \cdot \pi^{2 d} \cdot P^{2}\left(y, k_{1}, \ldots, k_{n}\right)+4(n+1)^{2}(\pi-3)^{2 N} \\
& <0+1=1 .
\end{aligned}
$$

Next suppose $F\left(y, x_{0}, \ldots, x_{n}\right) \leq 1$, where $y \in \mathbf{Z}$ and $\left(x_{0}, \ldots, x_{n}\right) \in \mathbf{R}^{n+1}$. There are integers $k_{i}$ such that $\left|\pi k_{i}-x_{i}\right| \leq \pi / 2(i=0, \ldots, n)$. The proof will be completed if we show that $P\left(y, k_{1}, \ldots, k_{n}\right)=0$, as it implies $y \in H$.

Since $x / 2 \leq 2 x / \pi \leq \sin x$ for every $x \in[0, \pi / 2]$, we obtain

$$
\frac{1}{2}\left|\pi k_{i}-x_{i}\right| \leq \sin \left|\pi k_{i}-x_{i}\right|=\left|\sin x_{i}\right| \quad(i=0, \ldots, n) .
$$

It follows from the condition $F\left(y, x_{0}, \ldots, x_{n}\right) \leq 1$ that

$$
\left|\sin x_{i}\right| \cdot\left|g_{i}\left(y, x_{0}, \ldots, x_{n}\right)\right| \leq \frac{1}{2(n+1)}(i=0, \ldots, n) .
$$

Applying the mean value theorem, we find $c_{0}, \ldots, c_{n} \in(-\pi / 2, \pi / 2)$ such that

$$
\begin{aligned}
\mid S\left(y, \pi k_{0}, \ldots, \pi k_{n}\right)- & S\left(y, x_{0}, \ldots, x_{n}\right) \mid \\
& =\left|\sum_{i=0}^{n} h_{i}\left(y, x_{0}+c_{0}, \ldots, x_{n}+c_{n}\right) \cdot\left(\pi k_{i}-x_{i}\right)\right| \\
& \leq \sum_{i=0}^{n} 2 \cdot g_{i}\left(y, x_{0}, \ldots, x_{n}\right) \cdot\left|\sin x_{i}\right| \leq 1,
\end{aligned}
$$

where the inequalities follow from Lemma 1, (3), and (4). Since

$$
S\left(y, x_{0}, \ldots, x_{n}\right) \leq 1 /\left(4(n+1)^{2}\right)<1,
$$

it follows from (5) that

$$
\left|S\left(y, \pi k_{0}, \ldots, \pi k_{n}\right)\right|<2
$$

and thus

$$
\begin{aligned}
\left(\pi k_{0}-3\right)^{2 N} & \leq Q^{2}\left(y, \pi k_{0}, \ldots, \pi k_{n}\right)+\left(\pi k_{0}-3\right)^{2 N} \\
& =S\left(y, \pi k_{0}, \ldots, \pi k_{n}\right)<2 .
\end{aligned}
$$

Now $k_{0}$ is an integer, therefore (7) can hold only if $k_{0}=1$. Consequently,

$$
\pi^{2 d} \cdot P^{2}\left(y, k_{1}, \ldots, k_{n}\right)=\pi^{2 d} Q^{2}\left(y, 1, k_{1}, \ldots, k_{n}\right)=Q^{2}\left(y, \pi k_{0}, \ldots, \pi k_{n}\right)<2
$$

by (11) and (6), and thus $P^{2}\left(y, k_{1}, \ldots, k_{n}\right)<1$. Since $P^{2}\left(y, k_{1}, \ldots, k_{n}\right)$ is an integer, we have $P^{2}\left(y, k_{1}, \ldots, k_{n}\right)=0$. 
Lemma 3. For every $x_{1}, \ldots, x_{n} \in \mathbf{R}, \delta>0$ and $K>0$ there exists a $t>K$ such that

$$
\left|x_{i}-t \cdot \sin t^{2 i}\right|<\delta \quad(i=1, \ldots, n) .
$$

Proof. We prove by induction on $n$. The case $n=1$ is clear. Suppose $n \geq 2$ and that the statement is true for $n-1$. We may assume $0<\delta<1$ and $K>1$. By the induction hypothesis there exists a $u>K+\left(\pi \cdot 2^{2 n+1} / \delta\right)+\left|x_{n}\right|$ such that

$$
\left|x_{i}-u \cdot \sin u^{2 i}\right|<\delta / 2 \quad(i=1, \ldots, n-1) .
$$

We put $v=u+\delta \cdot\left(8 n \cdot(u+1)^{2 n-2}\right)^{-1}$. Then we have, for every $t \in[u, v]$ and $i=1, \ldots, n-1$,

$$
\begin{aligned}
\left|t \cdot \sin t^{2 i}-u \cdot \sin u^{2 i}\right| & \leq\left|t \cdot\left(\sin t^{2 i}-\sin u^{2 i}\right)\right|+|t-u| \cdot\left|\sin u^{2 i}\right| \\
& \leq(u+1)\left(v^{2 i}-u^{2 i}\right)+\frac{\delta}{8} \\
& \leq(u+1) \cdot 2 i \cdot(u+1)^{2 i-1} \cdot \frac{\delta}{8 n(u+1)^{2 n-2}}+\frac{\delta}{8} \\
& <\frac{\delta}{4}+\frac{\delta}{8}<\frac{\delta}{2},
\end{aligned}
$$

and thus $\left|x_{i}-t \cdot \sin t^{2 i}\right|<\delta$. Since $u>\left|x_{n}\right|$ and

$$
v^{2 n} \geq u^{2 n}+2 n \cdot u^{2 n-1} \cdot \frac{\delta}{8 n(u+1)^{2 n-2}}>u^{2 n}+\frac{\delta}{4} \cdot \frac{u}{2^{2 n-2}}>u^{2 n}+2 \pi,
$$

we can find a $t \in[u, v]$ such that $t \cdot \sin t^{2 n}=x_{n}$.

Now we put

$$
f(y, x)=1-F\left(y, x \cdot \sin x^{2}, x \cdot \sin x^{4}, \ldots, x \cdot \sin x^{2 n+2}\right)
$$

for every $y \in \mathbf{Z}$ and $x \in \mathbf{R}$. Then $f(y, x) \in \mathcal{S}_{1}$ for every $y \in \mathbf{Z}$. We prove that

$$
\{y \in \mathbf{Z}: \exists x(f(y, x)>0)\}=H
$$

and

$$
\{y \in \mathbf{Z}: \exists x(f(y, x)=0)\}=H .
$$

Indeed, (8) and the inclusion $\{y \in \mathbf{Z}: \exists x(f(y, x)=0)\} \subset H$ are clear from Lemmas 2 and 3. Suppose $y \in H$. By (8) , there is a $u \in \mathbf{R}$ such that $f(y, u)>0$. Now we have $F \geq S \geq\left(x_{0}-3\right)^{2 N}$ everywhere, and thus

$$
f(y, x) \leq 1-\left(\left(x \cdot \sin x^{2}\right)-3\right)^{2 N}
$$

for every $x$. In particular, $f(y, 0)<0$. Therefore, we can find an $x$ with $f(y, x)$ $=0$, which proves (9). Since $H$ is not recursive, this completes the proof of Theorem 1.

\section{REFERENCES}

[1] B. F. Caviness, On canonical forms and simplification, Journal of the Association for computing Machinery 17 (2) (1970), 385-396. MR 43:7104

[2] L. Kuipers and H. Niederreiter, Uniform distribution of sequences. John Wiley \& Sons, 1974. MR 54:7415

[3] A. J. Macintyre, Exponential algebra. Logic and Algebra (Pontignano, 1994), 191-210. Lecture Notes in Pure and Applied Mathematics, No. 180. Dekker, 1996. MR 97h:03064 
[4] D. Richardson, Some undecidable problems involving elementary functions of a real variable, J. Symbolic Logic 33 (1968), 514-520. MR 39:1330

[5] P. S. Wang, The undecidability of the existence of zeros of real elementary functions, Journal of the Association for computing Machinery 21 (4) (1974), 586-589. MR 51:117

Department of Analysis, Eötvös Loránd University, Budapest, Pázmány Péter sétány 1/C 1117, Hungary

E-mail address: laczko@renyi.hu 